\title{
The Effectiveness of the Educational Therapeutic Program in the Treatment of Reading Difficulty for Pupils in the Primary Stage Arabic-Speaking.
}

\author{
Dr. Ladjal Yassine \\ Teacher of the conferences - University of Kasdi Marbeh Ouargla - Algeria
}

\begin{abstract}
The present study is interested in watching the impact of the skills of phonological treatment (processing) of the phonological consciousness and the memory of phonological work (phonological buckle (loop)) on the development of the mechanisms of the reader and its difficulties. The study was led on a total sample of 280 males and feminine pupils of the third and the fourth levels of the primary phase, their rate of age between $8-11$ years, including 50 children suffering from phonological dyslexia estimated by a test of reader aloud has lists of the words, the so called words and non-words. For that purpose, the researcher builds tools to measure the variables of the study to reach his goals, in particular both main objectives: the first one is to identify the relation between the phonological consciousness and the memory of phonological work with the reading on one hand and the dyslexia On the other hand, to determine the cause of the dyslexia and their contributions (taxes), the second is assured (insured) the efficiency of the program of treatment (processing) pedagogies for the dyslexia. In general, children with dyslexia have a great and persistent difficulty in phonological representations, explicit language units in conjunction with speech recognition skills. Resulted in our results supports the evolutionary hypothesis of the public deficit in the phonological processing skills, and concluded that awareness rhymes appear before tomography phonemic awareness and that of the child at this level of difficulty answering phoneme (vowel and consonants). In addition, multiple regression analysis shows that the decline in the phonological memory is the best indicator of dyslexia Arabic language weak phonological awareness. The studies also reached focus on the effectiveness of the proposed program for the treatment of specific phonological dyslexia and generally improve.
\end{abstract}

Key words: Dyslexia, phonological awareness, phonological memory, therapeutic protocol 